\title{
The response of cortical neurons to in vivo-like input current: theory and experiment: II. Time-varying and spatially distributed inputs
}

\author{
Michele Giugliano • Giancarlo La Camera • \\ Stefano Fusi · Walter Senn
}

Received: 16 July 2008 / Accepted: 2 October 2008

(c) Springer-Verlag 2008

\begin{abstract}
The response of a population of neurons to time-varying synaptic inputs can show a rich phenomenology, hardly predictable from the dynamical properties of the membrane's inherent time constants. For example, a network of neurons in a state of spontaneous activity can respond significantly more rapidly than each single neuron taken individually. Under the assumption that the statistics of the
\end{abstract}

M. Giugliano acknowledges support from the European Commission (FACETS and EUSynapse Project). G. La Camera is supported by the Intramural Research Program, NIMH. S. Fusi is supported by the Swiss National Science Foundation, grant PP00A-106556. W. Senn is supported by the Swiss National Science Foundation, grant no. 3152A0-105966. The views expressed in this article do not necessarily represent the views of the NIMH, NIH, or the United States Government.

M. Giugliano $(\varangle)$

Laboratory of Neural Microcircuitry, Ecole Polytechnique Fédérale de Lausanne, Station 15, 1015 Lausanne, Switzerland

e-mail: michele.giugliano@epfl.ch

\section{Giugliano}

Theoretical Neurobiology, University of Antwerp, Wilrijk, Belgium e-mail: michele.giugliano@ua.ac.be

\section{G. La Camera}

Laboratory of Neuropsychology,

National Institute of Mental Health, National Institutes of Health,

Bethesda, MD, USA

\section{S. Fusi}

Center for Theoretical Neuroscience, College of Physicians and Surgeons, Columbia University, New York, NY, USA

S. Fusi

Institute of Neuroinformatics, University of Zurich-ETH,

Zurich, Switzerland

W. Senn

Department of Physiology, University of Bern, Bern, Switzerland synaptic input is the same for a population of similarly behaving neurons (mean field approximation), it is possible to greatly simplify the study of neural circuits, both in the case in which the statistics of the input are stationary (reviewed in La Camera et al. in Biol Cybern, 2008) and in the case in which they are time varying and unevenly distributed over the dendritic tree. Here, we review theoretical and experimental results on the single-neuron properties that are relevant for the dynamical collective behavior of a population of neurons. We focus on the response of integrate-and-fire neurons and real cortical neurons to long-lasting, noisy, in vivo-like stationary inputs and show how the theory can predict the observed rhythmic activity of cultures of neurons. We then show how cortical neurons adapt on multiple time scales in response to input with stationary statistics in vitro. Next, we review how it is possible to study the general response properties of a neural circuit to time-varying inputs by estimating the response of single neurons to noisy sinusoidal currents. Finally, we address the dendrite-soma interactions in cortical neurons leading to gain modulation and spike bursts, and show how these effects can be captured by a two-compartment integrate-and-fire neuron. Most of the experimental results reviewed in this article have been successfully reproduced by simple integrate-and-fire model neurons.

Keywords Populations of spiking neurons - Dynamics . Integrate-and-fire model $\cdot$ Patch clamp $\cdot$ Calcium spikes

\section{Introduction}

Neurons in the intact cortex of the mammalian brain are driven by the synaptic current generated by thousands of other neurons. As their activity is determined by the spikes of large populations of neurons, it is important to study and 
characterize the collective properties of groups of cells. When similarly behaving neurons share approximately the same statistics of the synaptic current, these neurons can be grouped together, and they can be replaced by a single-representative cell. This approach, known as the "mean field approximation", is reviewed in La Camera et al. (2008) for the case in which (i) the statistics of the input current is stationary and (ii) the response of the neuron is quasi-static, i.e. it can be considered constant on a time interval of a few seconds. This approach can be extended to the more general case of time-varying statistics of the synaptic currents, and to the case where the output spike train is non-static. The extended approach is particularly important to describe transient, oscillatory and rhythmic neuronal activity of large populations of neurons, and it can predict dynamical behaviors that sometimes cannot be foreseen by looking at the passive properties of the neuronal membrane. Population response times can be very different from those predicted by the inherent time constants of every individual neuron and in general they depend on the full distribution of all dynamical variables characterizing the neuronal dynamics. A population of neurons with spontaneous activity will exhibit reaction times that are significantly shorter than the membrane time constant (see, e.g., van Rossum et al. 2002), because the depolarizations are distributed in the whole interval between the resting potential and the threshold for spike emission. As a consequence, some of the neurons are ready to generate an action potential quickly (order of $1 \mathrm{~ms}$ ) in response to a stimulus. Other single-neuron properties, like adaptation on time scales of hundreds of milliseconds to seconds, are also readily reflected by the population response. The extension of the mean field approach to include non-stationary properties are the focus of this review.

We start by presenting in some detail an approximated theoretical framework for the problem of predicting the dynamic response of a network of neurons to arbitrary timevarying stimuli (Renart et al. 2003; La Camera et al. 2004). As an application of this approach, we show how it is possible to make quantitative predictions on the rhythmic activity exhibited by networks of dissociated neurons (Giugliano et al. 2004). We then show how single neurons and populations of neurons exhibit firing rate adaptation on multiple time scales (La Camera et al. 2006), even in response to inputs with stationary statistics. Studies of the population response to fast-varying inputs are reviewed next, for which we are forced to abandon the approximated theoretical framework of the previous sections. In the case of fast-varying inputs, the full mathematical machinery of the so-called population density approach, based on the solution of a Fokker-Plank equation, would in general be needed (Knight 1972; Abbott and van Vreeswijk 1993; Treves 1993; Fusi and Mattia 1999; Brunel and Hakim 1999; Nykamp and Tranchina 2000). However, recent studies on simple integrate-and-fire (IF) model neurons revealed that it is possible to predict the population response to an arbitrary time-varying input by knowing the response of single neurons to the sinusoidal components that make the input signal (Brunel et al. 2001; Fourcaud and Brunel 2002). The single-neuron response can be estimated in the case of IF model neurons, or it can be measured directly for real cortical cells (Köndgen et al. 2008). Because of the features of sodium-mediated action potentials, cortical neurons are surprisingly good at relaying fast temporal information, while dynamical response attenuation and distortions affect the input-output transfer properties only for very fast $(\lesssim 5 \mathrm{~ms})$ input transients. In all these studies, IF neurons with firing rate adaptation and other small modifications could predict quantitatively many aspects of the behavior of real cortical neurons.

In the last section, we turn back to the static properties of the neuronal response function to show how it is modulated by the spatial input distributions along the dendritic tree. We review the experimental results that show how distal dendritic input can modulate in a nonlinear fashion the somatic stationary response function (Larkum et al. 2004). This modulation is due to an interaction between somatic action potentials and dendritic calcium spikes that under the right conditions can lead to a further burst of somatic action potentials (Larkum et al. 1999). Despite the complexity of these mechanisms, a simple somatic IF mechanism coupled with a dendritic compartment can capture the experimental phenomenology and explain the gain modulation induced by dentritic inputs. The extent to which simple spiking models can capture these complex phenomena and, thus, provide the field of neuroscience with a successful reductionist approach is further examined in Sect. 7.

\subsection{Response function of the leaky IF neuron}

Most of the material reviewed in this article is based on, or is related to, the response function of the adaptive leaky IF (LIF) neuron, reviewed in detail in the companion article (La Camera et al. 2008), to which the reviewer is referred for a more detailed exposition. We report here the main formulae for convenience. The LIF neuron is completely characterized by its membrane potential, $V$, which evolves according to

$$
\frac{\mathrm{d} V}{\mathrm{~d} t}=-\frac{V-V_{\text {rest }}}{\tau}+\frac{I}{C},
$$

until a threshold $\theta$ is reached. At this time, a spike is said to be emitted and $V$ is clamped to a reset potential $V_{\mathrm{r}}$ for a refractory period $\tau_{\mathrm{r}}$, after which motion resumes according to Eq. 1. $V_{\text {rest }}$ is the membrane's resting potential, $C$ is the membrane capacitance, $\tau=R C$, where $R$ is the membrane resistance, and $I$ is an input current. To emulate the noisy input current targeting neurons in vivo, the current $I$ is modeled as a stochastic process. In the diffusion approximation 
(Lánský and Sato 1999; Richardson and Gerstner 2005), the subthreshold dynamics of the membrane potential obeys the stochastic differential equation of the Ornstein-Uhlenbeck process (Tuckwell 1988):

$\mathrm{d} V=-\frac{V-V_{\text {rest }}}{\tau} \mathrm{d} t+\mu \mathrm{d} t+\sigma \xi_{t} \sqrt{\mathrm{d} t}$,

where

$\mu=m_{I} / C, \quad \sigma=\sqrt{2 \tau_{I}} s_{I} / C$

are the average and standard deviation in unit time of the membrane voltage, and $\xi_{t}$ is a Gaussian process with flat spectrum, zero average and unitary variance. $m_{I}$ and $s_{I}^{2}$ are the average and the variance of the synaptic input current, and $\sqrt{2 \tau_{I}}$ is a factor to preserve units.

The stationary response function of the model neuron defined by Eq. 2, with spike and reset mechanisms as specified above, is by definition the output firing rate as a function of (constant) $m_{I}$ and $s_{I}$, and is given by

$\Phi(\mu, \sigma ; \tau) \equiv\left[\tau_{\mathrm{r}}+\tau \int_{\hat{V}_{\mathrm{r}}}^{\hat{\theta}} \sqrt{\pi} \mathrm{e}^{u^{2}}(1+\operatorname{erf}(u)) \mathrm{d} u\right]^{-1}$,

where the "hat" operation applied to $\theta$ and $V_{\mathrm{r}}$ is defined by $\hat{z} \equiv(z-\mu \tau) / \sigma \sqrt{\tau}$. In the presence of firing rate adaptation, the adapted firing rate $f$ can be obtained as the self-consistent solution of

$f=\Phi\left(m_{I}-\alpha f, s_{I}\right)$,

where the parameter $\alpha$ quantifies the strength of adaptation. The derivation of Eq. 5 from a minimal model of firing rate adaptation is presented in detail in La Camera et al. (2008). A generalization to the time-dependent case is presented in the next section.

\section{Population response to arbitrary time-varying inputs}

In this section, we show how the single-neuron response function can be used to predict the dynamic behavior of networks of spiking neurons. This application goes beyond the stationary framework in which the mean field approach is derived, and provides us with an approximated solution of a complex Fokker-Plank equation in a two- or higher dimensional space (Brunel and Sergi 1998; Brunel and Hakim 1999; Moreno et al. 2002; Nykamp and Tranchina 2001; Fourcaud and Brunel 2002; Moreno-Bote and Parga 2004; Gigante et al. 2007a,b). We present a case study where the use of the stationary response function provides a good approximation to the full approach (Renart et al. 2003). This approximation can be extended to include firing rate adaptation, generalizing
Eq. 5 (La Camera et al. 2004), and is valid when the population dynamics is faster than the dynamics of single neurons. This allows one to assume that the population is always at equilibrium (i.e., the solution of the Fokker-Planck equation for which the derivative with respect to time is zero). This is typically true for adaptation, but in general the network dynamics is also faster than fast synaptic integration $(2-10 \mathrm{~ms})$, especially in the presence of large spontaneous activity (see, e.g., Renart et al. 2003; La Camera et al. 2008).

\subsection{Simplified mean field theory with time-varying inputs}

Consider an input spike train of time-varying frequency $v_{x}(t)$, targeting each cell of a population of neurons through $x$-receptor-mediated channels. Each spike contributes a postsynaptic current of the form $\bar{g}_{x} \mathrm{e}^{-t / \tau_{x}}$, where $\bar{g}_{x}$ is the peak conductance of the channels. In the diffusion approximation this produces an input current, $I_{x}$, which is an OrnsteinUhlenbeck process,

$\mathrm{d} I_{x}=-\frac{I_{x}-\bar{m}_{x}}{\tau_{x}} \mathrm{~d} t+\bar{s}_{x} \xi_{t} \sqrt{\frac{2 \mathrm{~d} t}{\tau_{x}}}$,

with $\bar{m}_{x}=\bar{g}_{x} v_{x}(t) \tau_{x}$ and $\bar{s}_{x}^{2}(t)=(1 / 2) \bar{g}_{x}^{2} v_{x}(t) \tau_{x}$ (La Camera et al. 2008). Renart et al. (2003) have suggested that the population activity of the network could be well predicted by

$f(t)=\Phi\left(m_{x}(t), s_{x}^{2}(t)\right)$

where $\Phi$ is the stationary response function (e.g., Eq. 4), and $m_{x}, s_{x}^{2}$ are the time-varying average and variance of $I_{x}$. These evolve according to the first-order dynamics $(\dot{y} \equiv \mathrm{d} y / \mathrm{d} t)$ :

$\tau_{x} \dot{m}_{x}=-\left(m_{x}-\bar{m}_{x}\right)$

and

$\frac{\tau_{x}}{2} \dot{s}_{x}^{2}=-\left(s_{x}^{2}-\bar{s}_{x}^{2}\right)$,

(e.g., see Gardiner, 1985). In this approximation, it is assumed that the network activity follows its time-varying input instantaneously. The reason for which this approximation works is that the typical reaction times of a population of integrate-and-fire neurons are short, of the order of a few milliseconds. This is mostly due to the fluctuations in the input current that distribute the depolarizations of the neurons over the whole range of permitted values. As a consequence, a non-negligible fraction of neurons is close to the threshold for spike emission, making the response of the network much faster than the response of any individual neuron (e.g., van Rossum et al. 2002). This justifies the assumption that the firing rate of the population is always at the steady state, despite an input with time-varying statistics $m_{x}(t), s_{x}(t)$, provided that the population dynamics are fast enough. 


\subsection{Extension to networks of adapting neurons}

The approach of the previous subsection can be extended to include firing rate adaptation. Consider the stationary, mean field model of the population adapted firing rate, Eq. 5, and write it as follows:

$f=\Phi\left(m_{x}-I_{\alpha}, s_{x}^{2}\right)$

$I_{\alpha}=\alpha f$

where $I_{\alpha}$, called the hyperpolarization (AHP) current, is a feedback current driven by the neuron's instantaneous output rate $f . I_{\alpha}$ can be derived to be of form $-\alpha f$ from a minimal cellular model of AHP current,

$\frac{\mathrm{d} I_{\alpha}}{\mathrm{d} t}=-\frac{I_{\alpha}}{\tau_{\alpha}}+\bar{g}_{\alpha} \sum_{k} \delta\left(t-t_{k}\right)$,

where the sum is over all spikes emitted by the neuron up to time $t$ and $\bar{g}_{\alpha}$ is the peak conductance. In the absence of actions potentials, $I_{\alpha}$ decays to zero with time constant $\tau_{\alpha}$, and $\alpha$ is linked to these cellular parameters by $\alpha=\bar{g}_{\alpha} \tau_{\alpha}$. This model works best in the presence of spontaneous activity (La Camera et al. 2008).

From Eq. 12 one can show that, in the case of slow variation of firing rate due to adaptation, $I_{\alpha}$ follows the neuron's own instantaneous firing rate with time constant $\tau_{\alpha}$ (La Camera et al. 2004):

$f=\Phi\left(m_{x}-I_{\alpha}, s_{x}^{2}\right)$

$\tau_{\alpha} \dot{I}_{\alpha}=-I_{\alpha}+\alpha f$,

Note that for a stationary stimulus, i.e. $v_{x}$ constant, after a transient of the order of $\max \left\{\tau_{x}, \tau_{\alpha}\right\}$, one recovers the stationary model Eqs. 10 and 11, with $m=\bar{m}_{x}, s=\bar{s}_{x}$.

In the case of several independent components, they follow their own synaptic dynamics and sum up in the argument of the response function to give the time-varying firing rate,

$f=\Phi\left(\sum_{x} m_{x}-I_{\alpha}, \sum_{x} s_{x}^{2}\right)$,

which replaces Eq. 13. This model is valid when the conditions for Eq. 7, of which it represents a generalization, hold.

Figure 1 shows the response of a population of uncoupled LIF neurons to a complex input, comprising an impulsive increase of $1 \mathrm{~ms}$ duration at $t=250 \mathrm{~ms}$ and a step increase at $t=400 \mathrm{~ms}$ (horizontal bars in Fig. 1) on top of the synaptic current. The synaptic current was made up of two fast components ( $\tau_{x}=5 \mathrm{~ms}$ ), one excitatory (AMPA-like), the other inhibitory $\left(\mathrm{GABA}_{A}\right.$-like), plus a third component mimicking slow (NMDA-like) current (with $\tau_{\text {nmda }}=100 \mathrm{~ms}$ ). The model makes a good prediction of the population activity, even during the fast transients. The small discrepancies are due to finite-size effects (Brunel and Hakim 1999; Mattia and Del Giudice 2002), and to the approximation used for

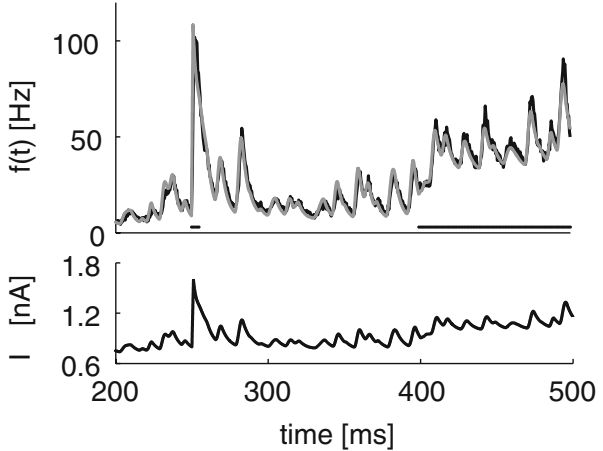

Fig. 1 Time-varying activity of a population of independent, adapting LIF neurons in response to a noisy, broadband stimulus. Top: prediction of the adapting rate model, Eqs. 13 and 14 (gray), compared to the simulations of 20,000 neurons (black). The activity after a transient of $200 \mathrm{~ms}$ is shown. The short horizontal bar indicates a pulse-like increase of $1 \mathrm{~ms}$ duration in the input current. The long horizontal bar indicates a step-like increase of both the excitatory and inhibitory input current. Bottom: Average time course of the stimulus (fluctuations around this average are not shown). See the text and La Camera et al. (2004) for details. Used with permission from La Camera et al. (2004). Copyright (c) 2004 by The MIT Press

the stationary response function [Eq. 4 corrected for fast correlated inputs (Brunel and Sergi 1998; Fourcaud and Brunel 2002)]. To be able to use this approximation, the synaptic current must be much faster than the membrane time constant, i.e.,

$\max _{x}\left\{\tau_{x}\right\} \ll \tau$.

Synaptic current with longer correlation times (e.g., NMDAlike or $\mathrm{GABA}_{B}$-like) can be approximated by a slowly changing current, i.e., their variance can be neglected in Eq. 15 (Brunel and Wang 2001).

\subsection{Further extensions}

The approach of this section can be extended to any model neuron whose response function, $\Phi$, is known, and not just to the LIF neuron Eq. 2 (see also Shriki et al. 2003). In particular, it can be extended to the conductance-based LIF neuron, with the caveat that condition Eq. 16 may not be fulfilled for currents with short time constants (e.g., AMPA-like and $\mathrm{GABA}_{A}$-like). This is because the effective time constant of the conductance-based neuron,

$\tau^{*}=\left(\frac{1}{\tau}+g_{e} v_{e}+g_{i} \nu_{i}\right)^{-1}$,

where $g_{e, i}, v_{e, i}$ are the excitatory (e) and inhibitory (i) input conductances and firing rates respectively, can reach values as small as a few ms, depending on the instantaneous firing rates. When condition Eq. 16 is not fulfilled, the approximated response function given by Moreno-Bote and Parga 

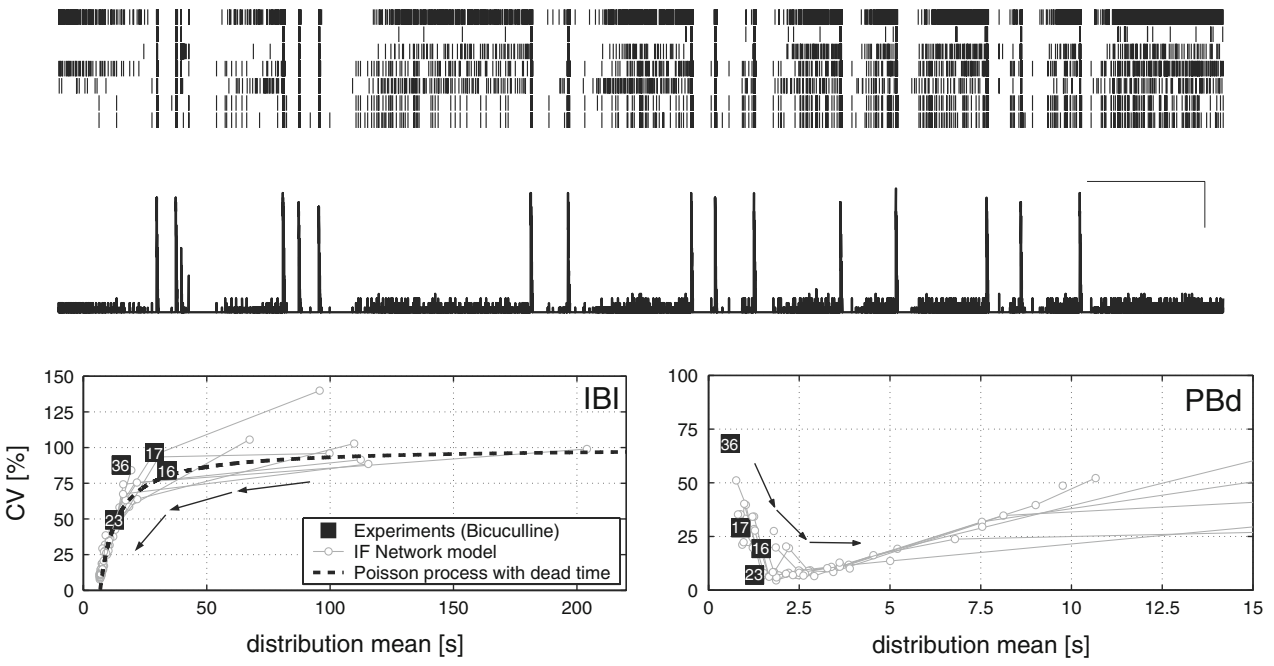

Fig. 2 Network activity emerging in dissociated cultures of neurons, detected by substrate arrays of extracellular microelectrodes (MEAs). The raster plot (upper panels) indicates the occurrence of spikes detected by seven substrate electrodes and, below, the resulting population firing rate (scale bars $60 \mathrm{~s}, 10 \mathrm{~Hz}$ ). Results from four MEAs experiments were compared to computer simulations of small recurrent networks of IF neurons, whose excitatory synaptic coupling was increased in the

(2005) should be used (see La Camera et al. 2004; La Camera et al. 2008 for details).

In the next section, we present an application of the theoretical framework developed in this section to the dynamics of rhythmic activity and population bursts.

\section{Rhythmic activity and population bursts}

Cultures of neurons from rat neocortex exhibit spontaneous, temporally patterned, network activity (Fig. 2). Neurons enzymatically dissociated from embryonic (rat) neocortex can be cultured in vitro and maintained under healthy conditions for up to several months in an incubator (Potter and DeMarse 2001). Neurons remain electrically active (Kamioka et al. 1996; Van den Pol et al. 1996), mature and continue developing ex vivo, and spontaneously reorganize into functional synaptic networks (Nakanishi and Kukita 1998) over the 2D surface of a Petri dish, or of an array of substrate micro-electrodes (Giugliano and Martinoia 2006). As opposed to brain slices, where spontaneous activity is largely abolished by the deafferentation following acute tissue cut, neuronal cultures spontaneously display a variety of collective spiking states (Wagenaar et al. 2006; Marom and Shahaf 2002). Thus, they offer a unique framework for identifying the response properties of individual neurons relevant to the collective dynamics of the whole network. In this section, we review the application of the mean field approach to the analysis of patterned rhythmic activity in those networks (Giugliano et al. 2004).

direction of arrows. Numbers associated with each markers help identifying the same experiment in both plots, reporting the coefficient of variation versus the mean, for the distribution of the interburst intervals (IBI) as well as for the population bursts (PBd). Used and modified with permission from Giugliano et al. (2004). Copyright $@ 2004$ by the American Physiological Society

3.1 Recurrent networks of IF neurons reproduce bursting activity

Small recurrent networks of 100-1,000 adaptive leaky IF excitatory neurons, incorporating the model parameters identified in the single-neuron response function experiments reviewed in La Camera et al. (2008) (see their Table 1), were able to capture the spontaneous rhythmic activity of Fig. 2 (van Vreeswijk and Hansel 2001; Ermentrout et al. 2001; Giugliano et al. 2004). To mimic the experimental conditions, where the action of inhibitory neurons is blocked by appropriate selective chemical antagonists (Giugliano et al. 2006), no inhibitory input was considered in the model network. The simulated network displayed spontaneously alternating intervals of asynchronous activity at low firing rate, and bursts of action potentials synchronized across the entire population (Fig. 3). To obtain this behavior, a modest background synaptic activity, meant to parallel the spontaneous release of neurotransmitter observed in mature and immature cultures, was sufficient.

Note that individual model neurons were not intrinsically bursting cells; instead, the synchronous emergence of brief epochs of intense firing rate was caused their recurrent synaptic interactions. Such epochs of intense activity were terminated by the progressive build up of spike-frequency adaptation in each IF neuron (Sect. 2.2, Eqs. 13-14). The spatial location, from which a network-wide synchronization event originates, varied randomly, consistent with the lack of a spatial structure in the network, and in keeping with the experimental findings (Fig. 2) (Kamioka et al. 1996; 


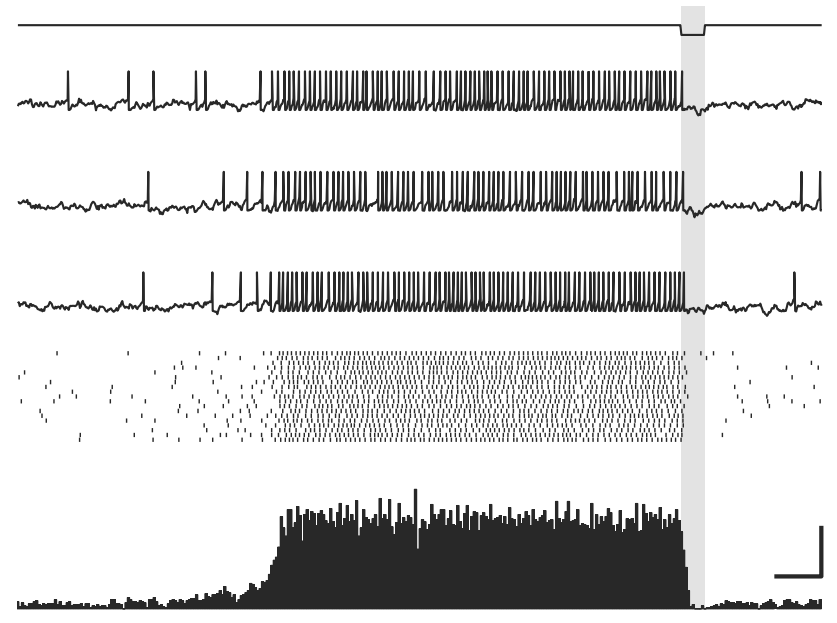

Fig. 3 A recurrent network, constituted by 100 excitatory identical IF model neurons, shows a spontaneous transition from a stable resting state $f_{\text {rest }}$ to another $f_{\text {burst }}$ due to the finite-size fluctuations. For the sake of illustration firing rate adaptation was not included (i.e., $\alpha=0$ in all neurons, see Eq. 5), so that $f_{\text {burst }}$ is a stable stationary state of the network (scale bars $200 \mathrm{~ms}$ and $25 \mathrm{~Hz} / 50 \mathrm{mV}$ ). The second transition $f_{\text {burst }} \rightarrow f_{\text {rest }}$ is induced by transiently reducing $m_{0}$ (upper continuous trace). Used and modified with permission from Giugliano et al. (2004). Copyright (C) 2004 by the American Physiological Society

Giugliano et al. 2004). Varying the average synaptic coupling strength and the overall number of synaptic connections mimicked different stages of in vitro synaptogenesis and development (Kamioka et al. 1996). For very weak coupling, the model network generated no synchronous burst of activity, in agreement to what observed during the first 5-7 days after plating (Giugliano et al. 2006). For larger but still weak coupling, the network generated rare and unpredictable population bursts which became more frequent and regular as soon as the excitatory synaptic coupling was increased (Fig. 2). The duration of the synchronized spiking epochs had comparable statistical properties in simulations and experiments (Giugliano et al. 2004).

\subsection{Mean field analysis of slow rhythmic activity}

In Fig. 3, the network flips between two states of activity either because of a strong enough finite-size fluctuation or because of the application of a transient stimulus. However, in the presence of firing rate adaptation it is possible to obtain an alternative sequence of flips between the two stable states without the application of transient stimuli. We show here how it is possible to predict this phenomenon by using the single-neuron response function in the mean field approach.

In the mean field approximation, the total synaptic current received by a generic model neuron is Gauss distributed, with its steady-state mean and variance given by

$m_{I}(f)=c N J f \tau+m_{0}, \quad s_{I}^{2}(f)=c N J^{2} f \tau / 2+s_{0}^{2}$.
Here, $f$ is the mean firing rate of the network, $N$ is the number of neurons of the network, $c$ is its "connectivity" (i.e., the average fraction of connections per neuron), and $J$ is the amplitude of the synaptic weights (Amit and Brunel 1997). $m_{0}$ and $s_{0}^{2}$ reflect the mean and variance of the current generated by spontaneous neurotransmitter release and other sources of randomness, assumed to be independent of $f$. The equilibria of the population dynamics can be identified as the self-consistent solution of the mean field equation (e.g., Amit and Brunel 1997; La Camera et al. 2008):

$f=\Phi\left(m_{I}(f), s_{I}(f)\right)$.

The fixed points at which the slope of the response function is less than 1 are stable.

For appropriate values of $J$, two stable equilibria can be found that we name here $f_{\text {rest }}$ and $f_{\text {burst }}$, with $f_{\text {rest }}<f_{\text {burst }}$ (see Fig. 4a, the curve with $J=12 \mathrm{pA}$ ). In the model network, spontaneous transitions from one of these stable states to the other can only occur due to fluctuations induced by finite-size effects (Brunel and Hakim 1999; Mattia and Del Giudice 2002), or triggered by an external stimulus (as in Fig. 3).

However, in the presence of firing rate adaptation, the state point $f_{\text {burst }}$ may destabilize after the neurons undergo adaptation (Fig. 4b). To understand why this may occur, note that in the presence of adaptation the fixed points of the network are given by the solution of the self-consistent equation

$f=\Phi\left(m_{I}(f)-\alpha f, s_{I}(f)\right)$,

(see Eq. 5). When $f$ is very small, like in state $f_{\text {rest }}$, the adapting term $-\alpha f$ is also small, and the properties of this state do not change appreciably. Things are different in the $f_{\text {burst }}$ state, however, as shown in Fig. 4b. Intuitively, while $f_{\text {rest }}$ remains a stable solution of Eq. 20, $f_{\text {burst }}$ is a solution only transiently, until adaptation has fully built up. This explains in simple terms the mechanisms behind the suppression of a single synchronized network event, or "population burst", and parallels the transient depression of firing seen e.g. in Fig. 2.

\section{Adaption over multiple time scales}

The simplified dynamic mean field theory of the previous sections could be extended to work in the presence of a single adaptation current of the AHP type, $I_{\alpha}$ (Sect. 2.2). This model of adaptation is general enough to describe most situations of interest (Benda and Herz 2003; La Camera et al. 2004). However, firing rate adaptation is a complex phenomenon occurring on several time scales and affected by different ion currents (Thorson and Biederman-Thorson 1974; Millhauser et al. 1988; Lowen and Teich 1992; Xu et al. 1996; Sah 1996; Schwindt et al. 1997; Powers et al. 1999; 


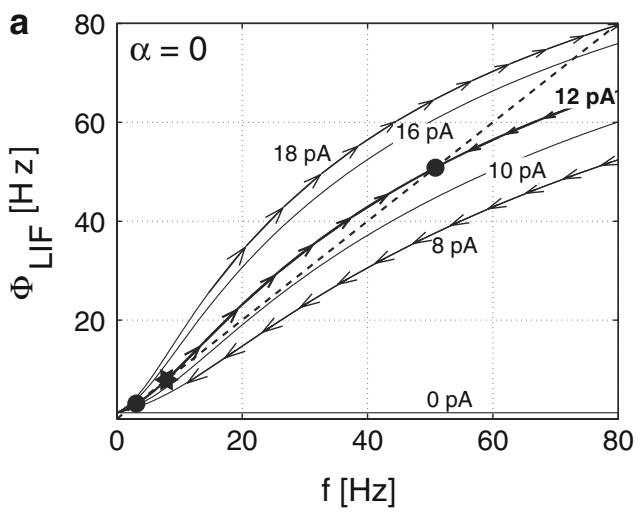

Fig. 4 Prediction of slow rhythmic activity from the properties of the single-neuron response function. Each curve is the firing rate of the population of LIF neurons $\left(\Phi_{\mathrm{LIF}}\right)$ as a function of the firing rate of the population itself $(f)$. When $\Phi_{\mathrm{LIF}}=f$, the network has reached a self-consistent state, which may be stable (closed circles) or unstable (star), depending on the slope of the response function at $f$. The slope of the response can be controlled by changing the value of the synaptic couplings $J$, both in the absence (a) and in the presence (b) of adaptation. a For $J=8$ or $10 \mathrm{pA}$, only the stable state at low firing rate is stable, named here $f_{\text {rest }}$. For $J=12 \mathrm{pA}$, a state with higher firing rate is also stable, taken here to represent a population burst (see the text). In these examples there is no firing rate adaptation $(\alpha=0$, see Eq. 5). b Same as $\mathbf{a}$ in the presence of firing rate adaptation (Eq. 5 with $\Phi=\Phi_{\mathrm{LIF}}$ ). The stability properties of the network change in the presence of adaptation: whereas $f_{\text {rest }}$ is stable also in the presence

Ulanovsky et al. 2004; Gilboa et al. 2005; Descalzo et al. 2005; Drew and Abbott 2006; La Camera et al. 2006; Wark et al. 2007). As a consequence, a single-adapting component may not be enough to describe the time course of cortical neurons, even in response to stationary input current (La Camera et al. 2006). Despite a large number of adapting processes acting on different time scales, an IF reduction of adapting spike trains could be obtained with not more than four independent adapting components in the case of pyramidal and fast-spiking (FS) neurons of the rat somatosensory cortex. These adapting processes acted on time scales ranging from a few ms to tens of seconds (Sect. 4.3). In pyramidal neurons, one of these components was found to facilitate the firing rate (i.e., to increase it) with a time constant of the order of $1 \mathrm{~s}$. In this section, we review the model used to capture the temporal aspect of the instantaneous firing rate in response to fluctuating inputs with stationary statistics.

\subsection{The generalized IF model with multiple adapting processes}

Recall that the model neuron having the quasi-stationary firing rate given by Eqs. 13 and 14 is described by a single variable, the membrane potential $V$, which below the spike threshold $\theta$ obeys Eq. 2 with $\mu=\left(m_{I}-I_{\alpha}\right) / C$, and $I_{\alpha}$ given by Eq. 12 .

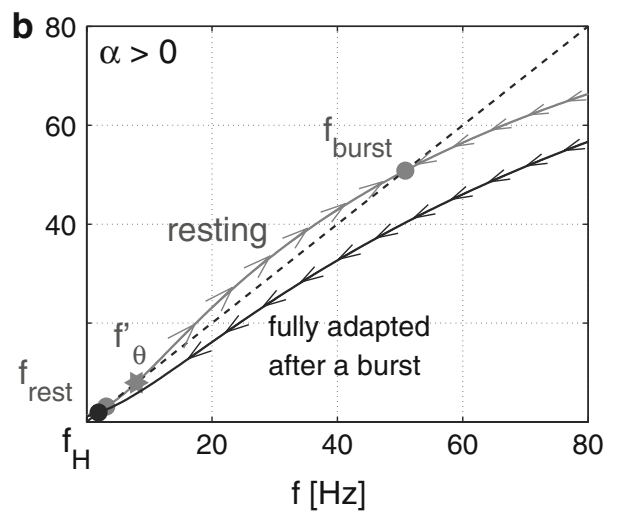

of adaptation, $f_{\text {burst }}$ destabilizes after the neurons undergo adaptation. Adaptation decreases the slope of the response function, which morphs into the lower curve, taking the network state into $f_{\text {rest }}$. In this state the neurons are negligibly adapted because of their low firing rate, and the response function becomes the upper curve again, where $f_{\text {burst }}$ is stable. Given the small basin of attraction of state $f_{\text {rest }}$ (this can be inferred from its distance, along the curve, from the unstable fixed point marked with 'asterisk'), an upward fluctuation of the spontaneous activity is sufficient to bring the network back into the $f_{\text {burst }}$ state. This state destabilizes after the neurons adapt, and the sequence of transitions repeats itself, with the network activity flipping between these two states in an activity-dependent way, as confirmed by simulations (not shown). Used and modified with permission from Giugliano et al. (2004). Copyright (C) 2004 by the American Physiological Society

A straightforward generalization of this model can be obtained by adding two or more independent components, $I_{\alpha}=\sum_{k} I_{\alpha_{k}}$, in Eq. 13 (La Camera et al. 2006). Each of the component processes obeys an equation like Eq. 12, with corresponding $\tau_{k}, \bar{g}_{k}$ such that $\bar{g}_{k} \tau_{k} \equiv \alpha_{k}$, and $\sum_{k} \alpha_{k}=\alpha$ (negative $\alpha_{k}$ s correspond to facilitating processes, i.e., process increasing the firing rate over time). This constraint ensures that the quasi-stationary firing rate of each spike train (reached when $t \gg \max _{k} \tau_{k}$ ) agrees with that given by the stationary solution in mean field, i.e., by the self-consistent solution of Eqs. 10 and 11.

\subsection{Reproducing the temporal response of cortical neurons}

The generalized adaptive model has been used to fit the time course of the firing rate of cortical neurons, defined as the inverse of the inter-spike interval (ISI) as a function of time, $f(t)=1 / \operatorname{ISI}(t)$, using a Monte carlo procedure (La Camera et al. 2006). The goodness-of-fit was then tested with $\chi^{2}$ test, using as the objective function the squared difference of the ISIs, i.e. $\chi_{\text {ISI }}^{2}=\sum_{j}\left(\operatorname{ISI}_{j}^{\exp }-\operatorname{ISI}_{j}^{\text {th }}\right)^{2}$. $\operatorname{ISI}^{\text {th }}(t)$ was obtained by simulating the full IF model described in the previous section. Note that it is necessary to consider the correct number of processes to estimate the time constants involved: since $\sum_{k=1}^{n} \bar{g}_{k} \tau_{k}=\alpha$, this constraint will be satisfied with different values of $\tau_{k}$ depending on $n$, the total number of processes. The peak conductances $\bar{g}_{k}$ were given by the ratio 

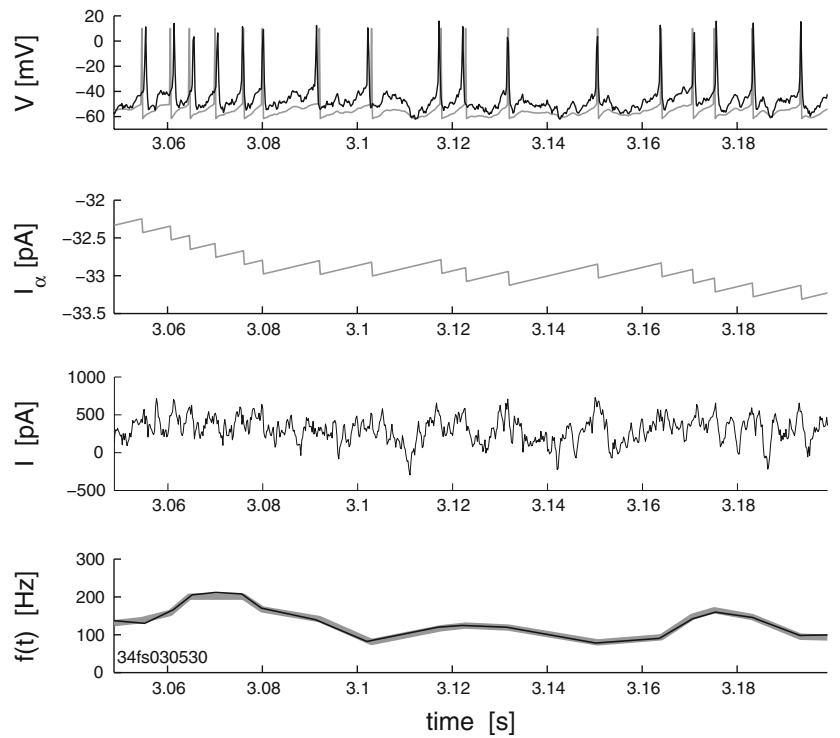

Fig. 5 Generalized adaptive LIF model and its best fit of the temporal response of a layer $5 \mathrm{FS}$ interneuron of rat somatosensory cortex. in each (panel) (from top to bottom, the membrane potential, the adaptation current (Eq. 12 with time constant $\tau_{\alpha}=2.2 \mathrm{~s}$ ), the total input current, and the instantaneous firing rate are shown (see the text). The input current was the same as injected into the neuron. In top and bottom panels, the model is in gray and the data are black. Used and modified with permission from La Camera et al. (2006). Copyright (c) 2006 by the American Physiological Society

$\alpha_{k} / \tau_{k}$ (negative if $\alpha_{k}<0$, representing facilitation instead of adaptation). An example showing the model with one component of adaptation and its best fit to an FS neuron of rat somatosensory cortex is shown in Fig. 5.

This procedure was not used to reproduce spike times with a millisecond precision (e.g., Jolivet et al. 2004, 2006), but only to capture the time course of the instantaneous firing rate.

\subsection{Time scales of temporal adaptation in cortical neurons}

In response to a stimulation of several seconds with fluctuating current with stationary statistics, the temporal response of FS interneurons of layers $2 / 3$ and layer 5 of rat somatosensory cortex could be captured with the generalized adaptive LIF neuron of Fig. 5 with only two components. The faster adapting process was of the order of $\sim 200 \mathrm{~ms}$; the slower had a broader distribution of time constants ranging from $\sim 1$ to $\sim 10 \mathrm{~s}$ (La Camera et al. 2006). Given that the maximal interval duration of stimulation was $10 \mathrm{~s}$, it is not excluded that even slower adapting processes could be present in these neurons.

Contrary to FS neurons, which typically had a consistent response to the same stimulus, pyramidal neurons of rat somatosensory cortex display a broader range of time constants and magnitude of the adapting processes involved. Four processes were identified according to the order of magnitude of their time constants, which were, from faster to slowest: a few milliseconds (affecting the first few ISIs only), $50-200 \mathrm{~ms}, 0.5-1 \mathrm{~s}$ (a facilitatory process), and order of seconds.

Despite the possibility to capture the temporal response of cortical neurons with only a handful of adapting processes, the distributions of time constants were broad, both within cells (pyramidal neurons) and across cells (pyramidal and FS neurons; see Table 3 of La Camera et al. 2006 for details). This variability in response to stimuli and across neurons suggests the possibility of a continuum of time scales, which may be the basis for scale-free adaptation (Fairhall et al. 2001b; Drew and Abbott 2006), a phenomenon with many potential computational consequences (Brenner et al. 2000; Fairhall et al. 2001a; Drew and Abbott 2006; Wark et al. 2007). Most of these computational consequences find application in response to stimuli with time-varying statistics; however, it is important to bear in mind that multiple adaptation processes are also observed in response to inputs with stationary statistics, as reviewed in this section.

\section{Response to sinusoidal inputs in the presence of fluctuations}

The dynamic mean field theory of the previous sections is a simplified approach which, in those cases where it can be applied, offers practical advantages to the study of the temporal evolution of the network activity in the presence of time-varying inputs. In the general case, the full population density approach should be used, but the equations are complex and are usually solved with perturbative techniques for the case of weak (but arbitrarily fast) input modulation. In this section, we reviewed some of the results obtained with this approach, which dates back to Knight (1972) and Treves (1993) (in the absence of noise), was generalized by Gerstner (2000), and has more recently been undertaken by Brunel and collaborators (Brunel et al. 2001; Fourcaud and Brunel 2002; Fourcaud-Trocmé et al. 2003; FourcaudTrocmé and Brunel 2005; see also Mattia and Del Giudice 2002).

The idea behind this approach is to analyze the response of the network to a weak oscillatory input (i.e., sinusoidal with frequency $\omega$ ),

$m_{I}=m_{0}+m_{1} \sin (2 \pi \omega t)$,

with $m_{1} \ll m_{0}$, in the presence of input fluctuations with constant amplitude $s_{I}$ (see e.g. Fig. 6a, b). The response of the network to this input can be found by solving perturbatively a Fokker-Planck equation in series of $1 / \omega$, assuming that the neuron's output firing rate is also sinusoidally modulated at the same frequency, 
$f(t)=f_{0}+f_{1}(\omega) \sin (2 \pi \omega t+\phi(\omega))$.

In networks of LIF neurons with instantaneous synapses, the amplitude of the response modulation $\left(f_{1}(\omega)\right)$ in the highfrequency regime is proportional to $1 / \sqrt{\omega}$, with a phase lag of 45 degrees (Brunel et al. 2001). In the presence of synaptic filtering, both the cut-off frequency and the phase lag are eliminated (Brunel et al. 2001). However, another important determinant to the response to oscillatory inputs, the mechanism for spike generation, is absent in the LIF neuron. For this reason, generalized models of IF neurons, where a fast transient response of the membrane potential above a given threshold can mimic an intrinsic mechanism of spike generation, were introduced and studied by Fourcaud-Trocmé et al. (2003) (called nonlinear IF models). These models are obtained by adding to the right hand side of Eq. 2 a term $\psi(V) / C$, which contains the nonlinear mechanism underlying the generation of an action potential, and a parameter $\Delta_{T}$ defining its sharpness. The firing rate modulation at high frequencies depends on $\psi$, being e.g. of the form $1 / \omega$ for an exponential function, and $1 / \omega^{2}$ for the quadratic case (Fourcaud-Trocmé et al. 2003). These results are also valid in the presence of conductance-based synaptic inputs, and suggest that in general the high-frequency modulation of the response follows a power law $\omega^{-\gamma}$, where the exponent $\gamma$ depends on the nonlinearity of the spike generating current $\psi(V)$ (Fourcaud-Trocmé et al. 2003). The (unmodulated) background synaptic noise also plays a role in the determination of the linear response (Brunel et al. 2001; Fourcaud and Brunel 2002), and so does the presence of firing rate adaptation (Fuhrmann et al. 2002).

These theoretical results provide a number of precise predictions which were tested experimentally by Köndgen et al. (2008) in rat cortical pyramidal neurons of the somatosensory cortex (Fig. 6). These authors found that the amplitude of the linear modulation $\left(f_{1}(\omega)\right)$ was generally constant and independent of $\omega$ up to rather high input frequencies, below a sharp cut-off of the order of 100-200 Hz. In this range, no phase-shift was observed other than what caused by spikefrequency adaptation at low frequencies (Fuhrmann et al. 2002) (Fig. 6c, d). This confirmed the role of the background synaptic noise in removing the resonances at multiples of the average firing rate response $f_{0}$ (Brunel et al. 2001; Fourcaud and Brunel 2002), allowing fast time-varying inputs to be encoded undistorted (i.e., without a phase lag). Beyond the cut-off frequency range, the high frequency linear response was found to decay as $f_{1}(\omega) \sim \omega^{-\gamma}$, with $\gamma>1$ and close to 2 , independently of the noise correlation time constant (Fig. 6c, d). This behavior is consistent with a nonlinear IF model with a very sharp spike, and a nonlinearity which is intermediate between exponential and quadratic (Fourcaud-Trocmé and Brunel 2005).
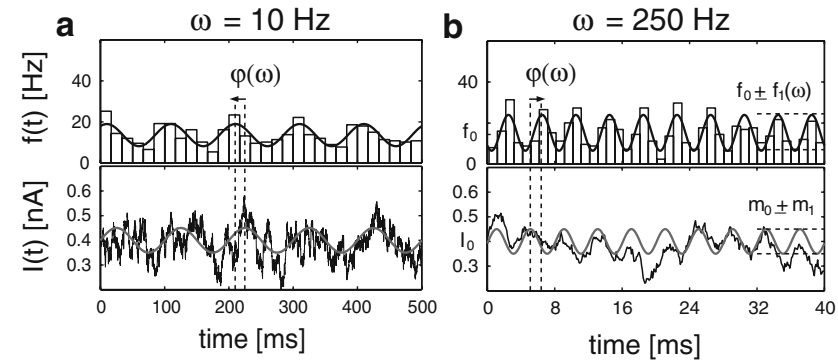

$\omega[\mathrm{Hz}]$

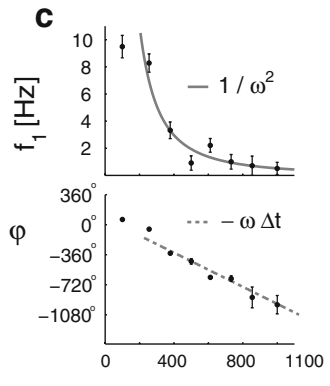

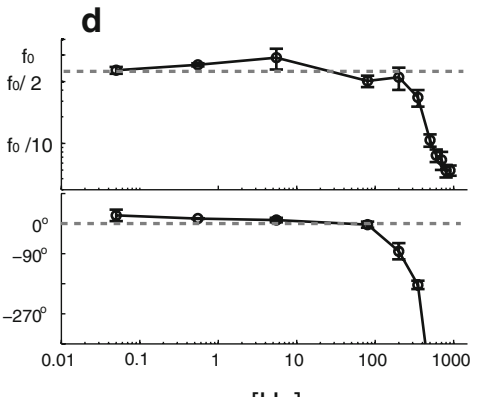

$\omega[\mathrm{Hz}]$
Fig. 6 Noisy sinusoidal input currents were injected in cortical neurons firing at 10-20 spikes/s to probe their dynamic response properties (a, b) and the impact of input fluctuations. The mean input was modulated as $m_{I}=m_{0}+m_{1} \sin (2 \pi \omega t)$, with $m_{1} \ll m_{0}$, while the amplitude of the background noise $\left(s_{I}\right)$ was kept constant. The neuron's output firing rate was also sinusoidally modulated at the same frequency $\omega$, $f(t)=f_{0}+f_{1}(\omega) \sin (2 \pi \omega t+\phi(\omega))$, over a wide range of input frequencies, $0-200 \mathrm{~Hz}$ (c single cell; d population). $f_{0}$ is the stationary response function (e.g., Eq. 4 for the case of the LIF neuron), $f_{1}$ is the response amplitude of the modulated response and $\phi$ is its phase shift. Used and modified with permission from Köndgen et al. (2008). Copyright (C) 2008, Oxford University Press

\section{Response to dendritic inputs and soma-dendritic interactions}

So far, we have been concerned with those properties of single neurons and network activity that could be analyzed assuming a point-neuron model. Point-neuron approximations may be a good description for small neurons with short and isotropic dendritic trees. However, the apical dendritic tree of layer 5 pyramidal neurons extends across all cortical layers with a length of roughly $1.5 \mathrm{~mm}$, and integrates inputs from different cortical and subcortical sources (Budd 1998; Binzegger et al. 2004; Oda et al. 2004). Whether the extended geometry of pyramidal neurons offers real computational advantages, or whether it only solves the 'packing problem' of collecting a large amount of synapses for a single integration process, remains an open issue and it will not be discussed here. Instead, we will focus on some of the phenomena that depend critically on such extended geometry and cannot be captured with point-neuron approximations, like the dendritically induced gain modulation of the somatic response and its control by inhibition (Larkum et al. 2004; Murayama et al. 2008). This will allow us to 
characterize the response of layer 5 pyramidal neurons to noisy input currents which are simultaneously injected in the soma and in the apical dendrite.

\subsection{Dendrites can differentiate between drivers, modulators and disruptors}

To structure the possible functional interactions among neurons, it has been suggested to classify neurons into drivers, modulators, and disruptors (Sherman and Guillery 1998). While drivers act additively on the output frequency, modulators act multiplicatively and disruptors block either of them. The multiplicative scaling of the neuronal response function is an efficient mechanism for modulating the neuronal responses in one cortical area by the activity in another area, which may be an important mechanism for cognitive processing (Salinas and Thier 2000).

It has been noted that a point neuron is able to integrate both drivers and modulators with the same type of ionotropic synapses (Chance et al. 2002; Abbott and Chance 2005). Here we show that an extended dendritic tree allows to incorporate all three operations by varying the location of the synaptic inputs (Fig. 7).

Experimental data and modeling studies confirm that excitatory synaptic input projecting to the proximal dendritic
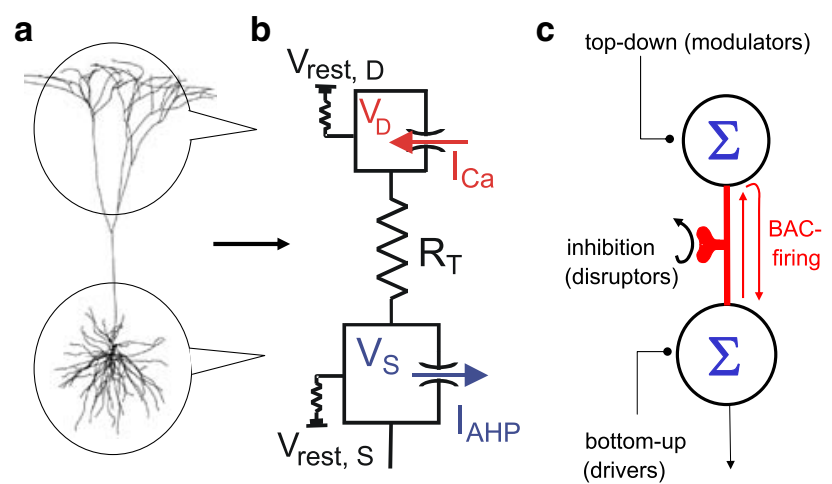

Fig. 7 Two-compartmental model of a cortical L5 pyramidal neuron. a Beside the basal integration zone around the soma (lower circle) L5 pyramidal neurons show an additional apical integration zone (upper circle) from where the signaling to the soma is mediated via dendritic calcium spike. b To capture the spatial input structure we consider a two-compartment neuron model with a voltage-dependent calcium current $\left(I_{\mathrm{Ca}}\right)$ in the dendritic compartment and a spike-triggered afterhyperpolarization current $\left(I_{\mathrm{Ca}}\right)$ in the somatic compartment. c Dendritic ('top-down') input increases the gain of the somatically ('bottom-up') induced current-to-rate response function via back-propagating action potential induced calcium (BAC) firing. This top-down input represents a multiplicative modulation of the somatic firing rate (with a factor $\geq 1$ ) which can be throttled by inhibitory dendritic input. Hence, the dendritic tree determines whether synaptic input either acts as 'driver', 'modulator', or 'disruptor' (Sherman and Guillery 1998), depending on whether it projects to the somatic region or the apical tuft, and whether it acts through excitation or inhibition tree can act efficiently as a driver (i.e., it shifts the response function to the left), while synaptic input to the distal dendritic tree can also act as a modulator (i.e., it also increases the gain of the response function), see Fig. 8a and Larkum et al. (2004). The basic mechanism allowing the distal dendritic input to modulate the gain relies on the generation of dendritic calcium spikes. These calcium spikes may be triggered by synaptic inputs on the distal dendritic tree impinging on a back-propagating action potential (AP). The calciuminduced dendritic depolarization propagates forward to the soma where it triggers one or several additional APs. Because this mechanism generates two or more APs out of one single AP, it represents a multiplicative operation on the response function. Since it is triggered by the joint emission of a backpropagating AP and a dendritic calcium spike, this mechanism is referred to as backpropagation-induced calcium (BAC) firing (see also Larkum et al. 1999).

In the experimental data, the multiplicative modulation is also accompanied by a left shift of the response function, which otherwise characterizes the action of drivers (Fig. 7). While in the experiment the left shift arises from a rather proximal positioning of the dendritic electrode, distal synaptic input further away from the soma will undergo a stronger attenuation and will barely contribute to the direct somatic depolarization. However, it will still contribute to the generation of a calcium spike in the apical dendrite and thus to a gain modulation of the somatic current-to-frequency response function.

Besides the action of somatic and dendritic excitation as drivers and modulators, respectively, inhibitory synaptic inputs efficiently operates as disrupters of both drivers and modulators. Inhibition may shunt the somatic voltage when the excitatory synaptic input projects proximally to the soma (Doiron et al. 2001; Mitchell and Silver 2003), or it may transiently block the calcium conductance and thus disrupt the gain modulation due to calcium spikes when the excitatory synaptic input projects to the distal dendrite (Larkum et al. 1999; Pérez-Garci et al. 2006).

\subsection{Two-compartmental IF model with dendritic calcium spikes}

The phenomena described in the previous subsection can be explained by an extension of the LIF point-neuron model to include two compartments, representing the somatic and the distal dendritic regions, respectively (Fig. 7a, b). A successful model of the calcium-induced dendritic depolarization requires the integration of some active dendritic current $\left(I_{\mathrm{Ca}}\right.$, Fig. 8c). To capture the fact that a dendritic calcium spike is only triggered by a fast voltage up-sweep across some voltage interval, a dynamic activation and inactivation of the calcium conductance must be considered (this can be done 
in terms of activation and inactivation variables, $m$ and $h$, respectively, see Larkum et al. 2004).

Because in the experiment the calcium-induced somatic AP bursts often cease even when the dendritic membrane is still depolarized, a potassium AHP current ( $I_{\mathrm{AHP}}$, see Eq. 12$)$ was included in the somatic compartment (Figs. 7b, 8b, c). This transiently activated leak current represents a sort of security valve, as it prevents the model neuron from being trapped in a calcium-induced depolarization plateau where it would continuously burst.

The two-compartmental IF model reproduces the firing rates of L5 pyramidal neurons in response to somatic and dendritic current injections (Fig. 8a). It also reproduces the increased gain of the somatic current-to-frequency curve in the presence of a dendritic background input (Fig. 8a, Larkum et al. 2004). When injecting a noisy somatic input current only, the individual spikes can be well predicted (Fig. 8b). However, when injecting a dendritic input currents, it is just the strong dendritic voltage deflection ('calcium spike') and the induced AP burst which can be predicted, but not the individual spike times within the burst (Fig. 8c). A detailed description of the two-compartmental model is provided in Larkum et al. (2004).

\subsection{Somatic response function for joint somatic and dendritic inputs}

In this section, we provide an approximated formula for the somatic response function of the two-compartmental IF model of the previous subsection. We start from an approximation of the cortical neuron response function observed in vitro (La Camera et al. 2008),

$f^{(1)}=\frac{(\bar{V}-\theta)}{\tau\left(\theta-V_{\text {reset }}\right)\left(1-\mathrm{e}^{-a(\bar{V}-\theta) / \sigma_{V}}\right)}$,

where $\tau=R C$ is the membrane time constant.

This formula was derived by Abbott and Chance (2005) using heuristic arguments related to the known behavior at large inputs, the effect of noise in the subthreshold versus the suprathreshold regime, and the often observed thresholdlinear behavior at rheobase of cortical neurons. The quantity $\bar{V}$ is the average of the membrane potential when the spiking generation mechanisms are inactivated, and $\sigma_{V}$ is its standard deviation. In terms of the mean, the standard deviation, and the correlation length $m_{I}, s_{I}$ and $\tau_{I}$, respectively, of the injected Ornstein-Uhlenbeck current (cf. Eq. 3) these quantities are expressed by

$\bar{V}=R m_{I}, \quad \sigma_{V}=R s_{I} \sqrt{2 \tau_{I} / \tau}$.

When Ornstein-Uhlenbeck currents are injected both into the soma and the distal dendrite, the corresponding somatic and dendritic voltages are characterized by $\bar{V}_{\mathrm{S}}, \sigma_{V_{\mathrm{S}}}$ and $\bar{V}_{\mathrm{D}}, \sigma_{V_{\mathrm{D}}}$,

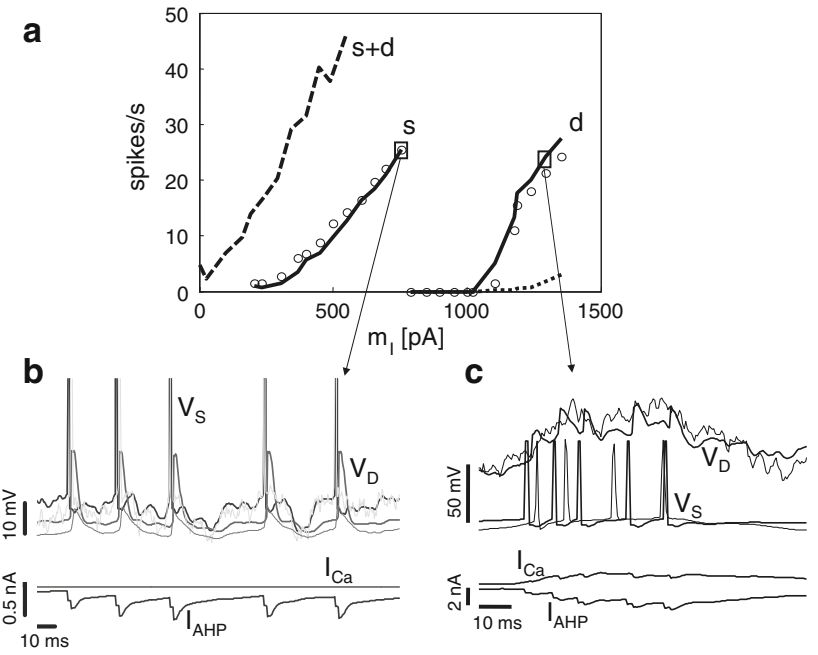

Fig. 8 Somatic and dendritic response functions and voltage traces for the two-compartmental model. a Firing rates of the model neuron in response to noisy input currents $\left(s_{I}=300 \mathrm{pA}\right)$ of varying mean $\left(m_{I}\right)$ injected individually in the soma (curve labeled by $s$; circles representing experimental data) and the dendritic compartment (curve labeled by $d$ ). When applying the same somatic currents in the presence of a noisy dendritic input (fixed to $m_{I}=750 \mathrm{pA}$ and $s_{I}=300 \mathrm{pA}$ ) the gain of the neuronal response function with respect to the somatic input is increased (dashed curve labeled by $s+d$ ). Blocking the calcium current in the dendrite would lead to a rather shallow response function for the dendritic current injection (dotted curve). b The somatic $\left(V_{\mathrm{S}}\right)$ and dendritic $\left(V_{\mathrm{D}}\right)$ model voltage trace in the case of pure somatic current injection closely predicts the experimental spike times. The thin smooth and noisy curves show the corresponding experimental traces. The lower panel confirms that no dendritic calcium current $\left(I_{\mathrm{Ca}}\right)$ is elicited in the model, whereas a strong after-hyperpolarization current $\left(I_{\mathrm{AHP}}\right)$ is triggered after each spike. $\mathbf{c}$ In the presence of a dendritic input, the dendritic calcium current $\left(I_{\mathrm{Ca}}\right)$ is responsible for the strong dendritic depolarization $\left(V_{\mathrm{D}}\right)$ generating the 'BAC-burst' $\left(V_{\mathrm{S}}\right)$. The parameters used in these simulations were (see Sect. 1.1 for a definition of the symbols, with $S$ standing for somatic compartment, and $D$ standing for dendritic compartment): $R_{\mathrm{S}}=R_{\mathrm{D}}=45 \mathrm{mV}, R_{\mathrm{T}}=70 \mathrm{mV}$, $V_{\text {rest, } \mathrm{S}}=-70 \mathrm{mV}, \quad V_{\text {rest,D }}=-60 \mathrm{mV}, \quad C_{\mathrm{S}}=\tau_{S} / R_{S}=13 / 45 \mathrm{nF}$, $C_{\mathrm{D}}=5 / 45 \mathrm{nF}$. The AHP current was as in Eq. 12, with the inclusion of a reversal potential of $-90 \mathrm{mV}$ and $g_{\alpha}=5 \mathrm{nS}, \tau_{\alpha}=60 \mathrm{~ms}$. The calcium current ( $I_{\mathrm{Ca}}$, see Larkum et al. 2004 for details) is specified by $g_{\mathrm{Ca}}=70 \mathrm{nS}, E_{\mathrm{Ca}}=120 \mathrm{mV}, m_{1 / 2}^{\infty}=0 \mathrm{mV}, h_{1 / 2}^{\infty}=-10 \mathrm{mV}$, $\operatorname{slope}\left(m^{\infty}\right)=1 / 3(\mathrm{mV})^{-1}, \operatorname{slope}\left(h^{\infty}\right)=1 / 5(\mathrm{mV})^{-1}, \tau_{m}=15 \mathrm{~ms}$ and $\tau_{h}=80 \mathrm{~ms}$. A somatic AP is elicited when $V_{S}$ crosses the threshold $\theta=-47 \mathrm{mV}$ and this activates an additional $I_{\mathrm{AHP}}$ component. Subsequently, $V_{\mathrm{S}}$ is clamped for $1 \mathrm{~ms}$ at $20 \mathrm{mV}$ and then reset for another $1 \mathrm{~ms}$ at $-52 \mathrm{mV}$, right $5 \mathrm{mV}$ below the threshold. To mimic the backpropagating AP, the dendritic voltage $V_{\mathrm{D}}$ is instantaneously raised by $20 \mathrm{mV}$ with a delay of $4 \mathrm{~ms}$ after $V_{S}$ crossed the threshold

respectively, each specified by the analog of the expressions in (24).

Recall that the induction of a dendritic calcium spike requires a fast dendritic voltage sweep crossing a certain voltage threshold. It is possible to trigger a calcium spike by dendritic input currents only, but in the presence of a backpropagating AP far less dendritic input is needed. We therefore concentrate on those calcium spikes which are triggered 
by the joint action of a back-propagating AP and a simultaneous dendritic voltage sweep. This will lead directly to a gain modulation of the somatic current-to-frequency response function (see Eq. 27).

The specific requirement on the voltage transient arises from the narrow window formed by the voltage-dependent activation and inactivation functions $\left(m_{\infty}\right.$ and $h_{\infty}$, respectively) which are typical for calcium currents (Koch 1999; Larkum et al. 2004). Since only a weak steady-state window current is possible with these gating functions, a slowly increasing dendritic voltage would merely inactivate the calcium conductance without being able to trigger a calcium spike. Dendritic voltage traces produced by an OrnsteinUhlenbeck process will therefore only lead to calcium spikes if the membrane potential deflections (characterized by $\sigma_{V_{\mathrm{D}}}$ ) are large, and especially if these deflections arise together with a dendritic steady-state depolarization $\left(\bar{V}_{\mathrm{D}}\right)$ which is strong compared to the AP threshold $(\theta)$. Hence, a criterium for the generation of a dendritic calcium spike given a somatically induced AP may have the form

$\sigma_{V_{\mathrm{D}}}\left(1+\bar{V}_{\mathrm{D}} / \theta\right) \geq \theta$,

where for the sake of simplicity we have chosen the same threshold $\theta$ for the generation of a calcium spike as for the generation of an AP. The probability, $P_{\mathrm{Ca}}$, of a dendritic calcium spike, conditioned on the earlier occurrence of a somatic AP within a short time interval, is a saturating, increasing function of the $V_{\mathrm{D}} / \theta$ term in Eq. 25; it can be expressed as

$P_{\mathrm{Ca}}=\left(1-\mathrm{e}^{-\left\lfloor\sigma_{V_{\mathrm{D}}}\left(1+\bar{V}_{\mathrm{D}} / \theta\right)-\theta\right\rfloor / b}\right) \rho$

where $b$ is a positive constant in units of voltage and $\rho$ is a scaling factor between 0 and 1 .

Next we consider the impact of the dendritic calcium spikes on the neuronal response function. The firing rate $f^{(2)}$ of the two-compartment model is due to the APs generated by the somatic current injection and the additional APs generated by BAC-firing. Each AP induces an iterative, albeit short-lived, process: a dendritic calcium spike occurring with probability $P_{\mathrm{Ca}}$ causes, on average, a subsequent $\mathrm{AP}$ at the soma, which in turn has a probability $P_{\mathrm{Ca}}$ to generate another $\mathrm{AP}$ due to a calcium spike, and so on. $f^{(2)}$ can then be obtained as the sum across all iteratively induced APs, starting with basic firing rate $f^{(1)}$ of the single compartment,

$f^{(2)}=f^{(1)} \sum_{i=0}^{\infty}\left(P_{\mathrm{Ca}}\right)^{i}=\frac{f^{(1)}}{1-P_{\mathrm{Ca}}}$,

where the right-hand-side is obtained by summing the geometric series. Alternatively, one may obtain $f^{(2)}$ from solving the recursion relation $f^{(2)}=f^{(1)}+f^{(2)} P_{\mathrm{Ca}}$. Plugging the expressions for $f^{(2)}$ and $P_{\mathrm{Ca}}$ (Eqs. 23 and 26, respectively) into the right-hand side of Eq. 27, one obtains the firing rate
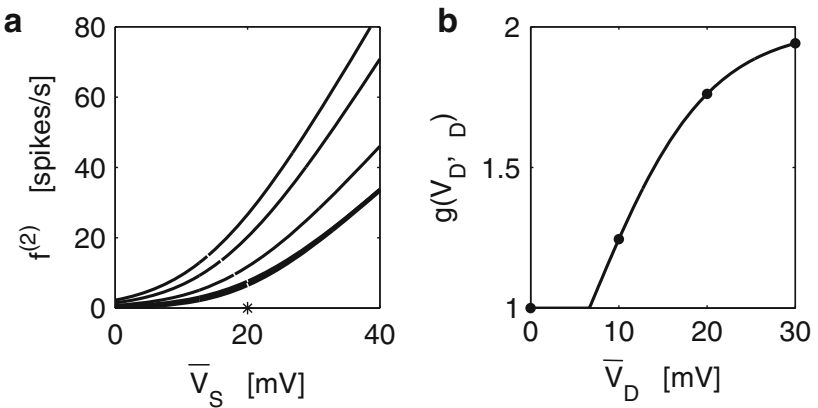

Fig. 9 Somatic response function for different dendritic inputs in the approximation of Sect. 6.3. a Firing rate $f^{(2)}$ for the two-compartment model (Eq. 28) as a function of the average somatic depolarization $\bar{V}_{\mathrm{S}}$, plotted for different values of the average dendritic depolarizations ( $\bar{V}_{\mathrm{D}}=0,10,20,30 \mathrm{mV}$ from bottom to top, with fixed $\sigma_{V_{\mathrm{S}}}=20 \mathrm{mV}$ and $\sigma_{V_{\mathrm{D}}}=15 \mathrm{mV}$ ). The star represents the voltage threshold $\theta=$ $20 \mathrm{mV}$. The lowest curve, representing the case $\bar{V}_{\mathrm{D}}=0$ (but with $\sigma_{V_{\mathrm{D}}}=15 \mathrm{mV}$ ), almost coincides with curve for pure somatic injection (Eq. 23). $\mathbf{b}$ The gain $g$ of the response functions in $\mathbf{a}$ as a function of average dendritic depolarization $\bar{V}_{\mathrm{D}}$ in the presence of a fixed noise amplitude $\sigma_{V_{\mathrm{D}}}=15 \mathrm{mV}$ (Eq. 29). The dots specify the values for $\bar{V}_{\mathrm{D}}$ and $g$ used to obtain the four curves in a. Other parameter values $a=5$, $b=5 \mathrm{mV}, \rho=0.5, \alpha=0.2, \tau=30 \mathrm{~ms}$

of the two-compartment IF model (see Fig. 9),

$$
\begin{aligned}
& f^{(2)}=\frac{g\left(\bar{V}_{\mathrm{D}}, \sigma_{\mathrm{D}}\right)\left(\bar{V}_{\mathrm{S}}-\theta\right)}{\tau\left(\theta-V_{\text {reset }}\right)\left(1-\mathrm{e}^{\left.-a\left(\bar{V}_{\mathrm{S}}-\theta\right) / \sigma_{V_{\mathrm{S}}}\right)},\right. \text { with }} \\
& g\left(\bar{V}_{\mathrm{D}}, \sigma_{\mathrm{D}}\right)=\frac{1}{(1-\rho)+\rho \mathrm{e}^{-\left\lfloor\sigma_{V_{\mathrm{D}}}\left(1+\bar{V}_{\mathrm{D}} / \theta\right)-\theta\right\rfloor / b}} .
\end{aligned}
$$

Expression (28) generalizes the one-compartmental response function (23) to the case of two compartments, where the second dendritic compartment acts as a gain modulator. The somatic and dendritic voltage variables $\bar{V}_{\mathrm{S}}, \sigma_{V_{\mathrm{S}}}$ and $\bar{V}_{\mathrm{D}}$, $\sigma_{V_{\mathrm{D}}}$, respectively, are related to the somatically and dendritically injected Ornstein-Uhlenbeck currents according to (24).

The two-compartment response function (28) neglects the passive propagation of the dendritic voltage toward the soma, and of the somatic voltage towards the dendrites. A passive dendro-somatic attenuation could be included in the model by substituting $V_{\mathrm{S}} \rightarrow V_{\mathrm{S}}+\alpha_{\mathrm{D}} V_{\mathrm{D}}$ and $\sigma_{\mathrm{S}} \rightarrow \sigma_{\mathrm{S}}+\alpha_{\mathrm{D}} \sigma_{\mathrm{D}}$, with $\alpha_{\mathrm{D}}$ representing a dendritic attenuation factor. The passive component would cause a left shift in the response function, whose strength is quantified by $\alpha_{\mathrm{D}}$. In the experiments, this factor appeared to be relatively large $(\sim 0.4)$. However, since in the experiments the dendritic electrode did not reach the calcium triggering zone at the apical bifurcation (Larkum et al. 2004), this factor may be much smaller in reality. Thus, the left shift of the response function induced by the passive dendritic input may be negligible compared to the induced gain increase (cf. Fig. 9a). 


\section{Discussion}

Large populations of neurons have a large number of degrees of freedom, giving rise to very rich and complex collective dynamics. In the network models reviewed in this article, the study of such a rich behavior can be simplified, because it can be reduced to the study of the time development of the distribution of the variables that characterize the singleneuron dynamics (e.g., the depolarization in the case of IF models). However, even with such a simplification, the equations governing the network dynamics are still difficult to solve. For example, in the case of weak enough synaptic interactions, the equation governing the distribution of the depolarizations of IF neurons is a Fokker-Planck equation. Although the dimensionality of the equation is low (the only dynamical variable is the depolarization), the boundary conditions, corresponding to the threshold for emitting a spike and to the lower bound of the depolarization, make it extremely difficult to find a general analytical solution. The only exact solutions known can be obtained under the assumption that the statistics of the synaptic input are stationary (La Camera et al. 2008). For simple IF models, however, it is possible to simplify further the study of the timedependent network dynamics in at least two situations: (1) when the relaxation dynamics of the Fokker-Planck equation are significantly faster than the time scale over which the input varies (as in the case of slow synaptic currents), and (2) when the amplitude of the modulations of the input statistics are small (perturbation theory). In both cases, we have shown here that it is possible to reduce the study of neural populations to the analysis of the response of single neurons to noisy inputs. This approach is possible also in the case of real neurons, for which the responses can be measured in experiments.

What could these experiments reveal that was not known for the neuronal model used to develop the theory? The experiments provided us with an estimate of the parameters of the neuron model, which is crucial for quantitative modeling studies. However, there are at least two other important, unexpected results that emerged from the analysis of the experimental measurements and that turned out to be independent of the specific neuronal model that was used to design the experiment itself. The first result is that populations of real neurons respond fast (Köndgen et al. 2008), significantly faster not only than any single neuron (which was expected), but also faster than predicted by simple IF models. The linear response to sinusoidal currents is not attenuated up to frequencies of a few hundreds $\mathrm{Hz}$. The second unexpected result is related to adaptation on multiple time scales. A quantitative analysis of the response of rat pyramidal and fast-spiking neurons to long lasting, noisy stimuli with stationary statistics revealed that the activity of cortical neurons is modulated over multiple time scales ranging from hundreds of milliseconds to seconds. The response of the neurons could be modeled with IF neurons with multiple mechanisms of adaptation and facilitation. Every neuron had up to four mechanisms operating on different time scales. Moreover, the time scales varied widely from neuron to neuron, allowing a population to show responses on almost a continuum of time scales.

In the second part of this review, we have considered the single-neuron response to inputs that are distributed on the dendritic tree. The simplicity of the IF model contrasts with the complexity of the dendritic arborization of some pyramidal neurons, with their regenerative membrane currents and clustered synaptic inputs (Spruston 2008). The extended geometry of cortical neurons and their nonlinear dentritic properties may offer additional computational power by exploiting nonlinear dendritic properties (Poirazi et al. 2003; Polsky et al. 2004), like the multiplicative gain modulation of the somatic response function reviewed here.

The gain of the response function can be modulated by various mechanisms, e.g., through the action of neuromodulators (Zhang and Arsenault 2005; Thurley et al. 2008), the strength of after-hyperpolarization currents (Higgs et al. 2006), or a balanced change of noisy excitation and inhibition (Hô and Destexhe 2000; Destexhe et al. 2001; Doiron et al. 2001; Chance et al. 2002; Longtin et al. 2002; Mitchell and Silver 2003). These forms of gain modulation are implementable in point neurons, and were not considered here. Other forms require instead multi-compartmentalization, like those arising from passive dendritic integration (Prescott and De Koninck 2003; Mehaffey et al. 2005; Capaday and van Vreeswijk 2006).

We reviewed a dendritically controlled gain modulation mediated by BAC firing and dendritic calcium spikes. This phenomenon relies on regenerative dendritic calcium conductances and can be tuned by acting directly on those conductances (Larkum et al. 2004), e.g. by selectively blocking them through activation of dendritic $\mathrm{GABA}_{A}$ and $\mathrm{GABA}_{B}$ receptors (Pérez-Garci et al. 2006). These tuning options extend the functionality of pyramidal neurons by allowing them to distinguish between synaptic drivers, modulators and disruptors (see Sherman and Guillery 1998 and Fig. 7c). Recent experimental evidence from awake rats suggests that these different types of inputs may also play a functional role in vivo (Murayama et al. 2008).

The mechanism of BAC-firing and BAC-firing-induced gain modulation is related to a specific type of neural code, a burst-timing code, which is different from both firing rate codes (e.g., of Figs. 1, 8a) and spike-timing codes. A calcium spike, triggered by the joint occurrence of a back-propagating $\mathrm{AP}$ and the dendritic input, elicits in turn several consecutive sodium spikes at the soma within roughly $30 \mathrm{~ms}$ (Larkum et al. 1999). This mechanism allows a coincidence signalling to downstream neurons. Since inputs to the soma and 
the apical tree may originate from different cortical sources (Budd 1998; Oda et al. 2004), burst-timing could provide a way to detect and signal the coincident occurrence of bottomup and top-down signals.

While in the present review we have focussed on the current-to-frequency transfer function and its modulation by temporal and spatial inputs, other aspects of the neuronal integration determine the encoding of synaptic inputs. Among these are the spatial distribution of inputs along dendrites as pioneered by Rall (1967), nonlinear dendritic interactions (for reviews see Koch and Segev 2000, Segev and London 2000), or the impact of high conductance states (Destexhe et al. 2003; Geisler et al. 2005).

For us, the ability of simplified models to capture much of the rich and varied experimental phenomenology of cortical neurons embedded in an in vivo-like environment indicates the success of the reductionist approach in neurophysiology. We also hope that recent observations, like gain modulation by distal dendritic inputs or the divergence of the response functions in prefrontal cortex neurons (Arsiero et al. 2007), can open the door for new quantitative models and their application to analysis of network behavior. While such an interaction between theory and experiment is a widely consolidated tradition in physics, it is becoming only slowly established in neuroscience.

Acknowledgments We thank M. Arsiero, P. Darbon, H. Köndgen, M. E. Larkum, H.-R. Lüscher and A. Rauch for their many contributions to the experimental characterization of the response function of neocortical neurons. Many of the ideas and the vision behind the work described in this article were pioneered by Prof. Daniel Amit. This work is dedicated to his memory.

\section{References}

Abbott L, Chance F (2005) Drivers and modulators from push-pull and balanced synaptic input. Prog Brain Res 149:147-155

Abbott L, van Vreeswijk C (1993) Asynchronous states in networks of pulse-coupled oscillators. Phys Rev E 48:1483-1490

Amit D, Brunel N (1997) Model of global spontaneous activity and local structured (learned) delay activity during delay. Cereb Cortex 7:237-252

Arsiero M, Lüscher HR, Lundstrom B, Giugliano M (2007) The impact of input fluctuations on the frequency-current relationships of layer 5 pyramidal neurons in the rat medial prefrontal cortex. J Neurosci 27:3274-3284

Benda J, Herz A (2003) A universal model for spike-frequency adaptation. Neural Comput 15:2523-2564

Binzegger T, Douglas R, Martin K (2004) A quantitative map of the circuit of cat primary visual cortex. J Neurosci 24:8441-8453

Brenner N, Bialek W, de Ruyter van Steveninck R (2000) Adaptive rescaling maximizes information transmission. Neuron 26(3): 695-702

Brunel N, Hakim V (1999) Fast global oscillations in networks of integrate-and-fire neurons with low firing rates. Neural Comput 11:1621-1671
Brunel N, Sergi S (1998) Firing frequency of leaky integrate-and-fire neurons with synaptic currents dynamic. J Theor Biol 195:87-95

Brunel N, Wang XJ (2001) Effects of neuromodulation in a cortical network model of object working memory dominated by recurrent inhibition. J Comput Neurosci 11:63-85

Brunel N, Chance FS, Fourcaud N, Abbott LF (2001) Effects of synaptic noise and filtering on the frequency response of spiking neurons. Phys Rev Lett 86(10):2186-2189

Budd J (1998) Extrastriate feedback to primary visual cortex in primates: a quantitative analysis of connectivity. Proc R Soc Lond B Biol Sci 265(1):1037-1044

Capaday C, van Vreeswijk C (2006) Direct control of firing rate gain by dendritic shunting inhibition. J Integr Neurosci 5(2):199-222

Chance F, Abbott L, Reyes A (2002) Gain modulation from background synaptic input. Neuron 35:773-782

Descalzo V, Nowak L, Brumberg J, McCormick D, Sanchez-Vives M (2005) Slow adaptation in fast spiking neurons in visual cortex. J Neurophysiol 93:1111-1118

Destexhe A, Rudolph M, Fellous JM, Sejnowski TJ (2001) Fluctuating dynamic conductances recreate in-vivo like activity in neocortical neurons. Neuroscience 107:13-24

Destexhe A, Rudolph M, Paré D (2003) The high-conductance state of neocortical neurons in vivo. Nat Rev Neurosci 4(3):739-751

Doiron B, Longtin A, Berman N, Maler L (2001) Subtractive and divisive inhibition: effect of voltage-dependent inhibitory conductances and noise. Neural Comput 13(1):227-248

Drew P, Abbott L (2006) Models and properties of power-law adaptation in neural systems. J Neurophysiol 96:826-833

Ermentrout B, Pascal M, Gutkin B (2001) The effects of spike frequency adaptation and negative feedback on the synchronization of neural oscillators. Neural Comput 13(6):1285-1310

Fairhall A, Lewen G, Bialek W, de Ruyter van Steveninck R (2001a) Efficiency and ambiguity in an adaptive neural code. Nature 412:787-792

Fairhall A, Lewen G, Bialek W, de Ruyter van Steveninck R (2001b) Multiple timescales of adaptation in a neural code. In: Leen TK, Dietterich TG, Tresp V (eds) Advances in neural information processing systems, vol 13. MIT Press, Cambridge, pp 124-130

Fourcaud N, Brunel N (2002) Dynamics of the firing probability of noisy integrate-and-fire neurons. Neural Comput 14:2057-2110

Fourcaud-Trocmé N, Brunel N (2005) Dynamics of the instantaneous firing rate in response to changes in input statistics. J Comput Neurosci 18(3):311-321

Fourcaud-Trocmé N, Hansel H, van Vreeswijk C, Brunel N (2003) How spike generation mechanisms determine the neuronal response to fluctuating inputs. J Neurosci 23:11,628-11,640

Fuhrmann G, Markram H, Tsodyks M (2002) Spike frequency adaptation and neocortical rhythms. J Neurophysiol 88:761-770

Fusi S, Mattia M (1999) Collective behavior of networks with linear (VLSI) integrate and fire neurons. Neural Comput 11:633-652

Gardiner CW (1985) Handbook of stochastic methods. Springer, Heidelberg

Geisler C, Brunel N, Wang X (2005) Contributions of intrinsic membrane dynamics to fast network oscillations with irregular neuronal discharges. J Neurophysiol 94(6):4344-4361

Gerstner W (2000) Population dynamics of spiking neurons: fast transients, asynchronous states, and locking. Neural Comput 12:43-90

Gigante G, Del Giudice P, Mattia M (2007a) Frequency-dependent response properties of adapting spiking neurons. Math Biosci 207:336-351

Gigante G, Mattia M, Del Giudice P (2007b) Diverse populationbursting modes of adapting spiking neurons. Phys Rev Lett 98:148,101

Gilboa G, Chen R, Brenner N (2005) History-dependent multiple-timescale dynamics in a single-neuron model. J Neurosci 25(28):64796489 
Giugliano M, Martinoia S (2006) Substrate arrays of microelectrodes for in itro electrophysiology. In: Wiley encyclopedia of biomedical engineering. Wiley, Hoboken

Giugliano M, Darbon P, Arsiero M, Lüscher HR, Streit J (2004) Singleneuron discharge properties and network activity in dissociated cultures of neocortex. J Neurophysiol 92:977-996

Giugliano M, Arsiero M, Darbon P, Streit J, Lüscher HR (2006) Emerging network activity in dissociated cultures of neocortex: novel electrophysiological protocols and mathematical modelling. In: Advances in network electrophysiology using multi-electrode arrays. Kluwer, New York

Higgs M, Slee S, Spain W (2006) Diversity of gain modulation by noise in neocortical neurons: regulation by the slow afterhyperpolarization conductance. J Neurosci 26(34):8787-8799

Hô N, Destexhe A (2000) Synaptic background activity enhances the responsiveness of neocortical pyramidal neurons. J Neurophysiol 84(3): 1488-1496

Jolivet R, Lewis T, Gerstner W (2004) Generalized integrate-and-fire models of neuronal activity approximate spike trains of a detailed model to a high degree of accuracy. J Neurophysiol 92:959-976

Jolivet R, Rauch A, Lüscher HR, Gerstner W (2006) Predicting spike timing of neocortical pyramidal neurons by simple threshold models. J Comput Neurosci. doi:10.1007/s10827-006-7074-5

Kamioka H, Maeda E, Jimbo Y, Robinson H, Kawana A (1996) Spontaneous periodic synchronized bursting during formation of mature patterns of connections in cortical cultures. Neurosci Lett 206:109-112

Knight BW (1972) Dynamics of encoding of a populations of neurons. J Gen Physiol 59:734-736

Koch C (1999) Biophysics of computation. Oxford University Press, New York

Koch C, Segev I (2000) The role of single neurons in information processing. Nat Neurosci Suppl 3:1171-1177

Köndgen H, Geisler C, Fusi S, Lüscher HR, Wang XJ, Giugliano M (2008) The dynamical response properties of neocortical neurons to temporally modulated noisy inputs in vitro. Cereb Cortex 18(9):2086-2097

La Camera G, Rauch A, Senn W, Lüscher HR, Fusi S (2004) Minimal models of adapted neuronal response to in vivo-like input currents. Neural Comput 16:2101-2124

La Camera G, Rauch A, Thurbon D, Lüscher HR, Senn W, Fusi S (2006) Multiple time scales of temporal response in pyramidal and fast spiking cortical neurons. J Neurophysiol 96:3448-3464

La Camera G, Giugliano M, Senn W, Fusi S (2008) The response function of cortical neurons: theory and experiment: I. Noisy inputs with stationary statistics. Biol Cybern 99. doi:10.1007/ s00422-008-0272-7

Lánský P, Sato S (1999) The stochastic diffusion models of nerve membrane depolarization and interspike interval generation. J Peripher Nerv Syst 4:27-42

Larkum M, Senn W, Lüscher H (2004) Top-down dendritic input increases the gain of layer 5 pyramidal neurons. Cereb Cortex 14:1059-1070

Larkum ME, Zhu JJ, Sakmann B (1999) A new cellular mechanism for coupling inputs arriving at different cortical layers. Nature 398:338-341

Longtin A, Doiron B, Bulsara A (2002) Noise-induced divisive gain control in neuron models. Biosystems 67:147,156

Lowen SB, Teich MC (1992) Auditory-nerve action potentials form a nonrenewal point process over short as well as long time scales. J Acoust Soc Am 92(2 Pt 1):803-806

Marom S, Shahaf G (2002) Development, learning and memory in large random networks of cortical neurons: lessons beyond anatomy. Q Rev Biophys 35:63-87

Mattia M, Del Giudice P (2002) Population dynamics of interacting spiking neurons. Phys Rev E 66:051,917
Mehaffey W, Doiron B, Maler L, Turner R (2005) Deterministic multiplicative gain control with active dendrites. J Neurosci 26(43):9968-9977

Millhauser GL, Salpeter EE, Oswald RE (1988) Diffusion models of ion-channel gating and the origin of power-law distributions from single-channel recording. Proc Natl Acad Sci USA 85(5):15031507

Mitchell S, Silver R (2003) Shunting inhibition modulates neuronal gain during synaptic excitation. Neuron 38(1):433-445

Moreno R, de la Rocha J, Renart A, Parga N (2002) Response of spiking neurons to correlated inputs. Phys Rev Lett 89:288,101

Moreno-Bote R, Parga N (2004) Role of synaptic filtering on the firing response of simple model neurons. Phys Rev Lett 92:028,102

Moreno-Bote R, Parga N (2005) Membrane potential and response properties of populations of cortical neurons in the high conductance state. Phys Rev Lett 94:088,103

Murayama M, Prez-Garci E, Nevian T, Bock T, Senn W, Larkum M (2008) Dendritic encoding of sensory stimuli controlled by deep cortical interneurons. Nature (in press)

Nakanishi K, Kukita F (1998) Functional synapses in synchronized bursting of neocortical neurons in culture. Brain Res 795(1,2):137-146

Nykamp D, Tranchina D (2001) A population density approach that facilitates large-scale modeling of neural networks: extension to slow inhibitory synapses. Neural Comput 13:511-546

Nykamp DQ, Tranchina D (2000) A population density approach that facilitates large-scale modeling of neural networks: analysis and an application to orientation tuning. J Comput Neurosci 8:1930

Oda S, Kishi K, Yang J, Chen S, Yokofujita J, Igarashi H, Tanihata S, Kuroda M (2004) Thalamocortical projection from the ventral posteromedial nucleus sends its collaterals to layer I of the primary somatosensory cortex in rat. Neurosci Lett 367:394-398

Pérez-Garci E, Gassmann M, Bettler B, Larkum M (2006) The gabab1b isoform mediates long-lasting inhibition of dendritic $\mathrm{Ca} 2+$ spikes in layer 5 somatosensory pyramidal neurons. Neuron 18(50):603616

Poirazi P, Brannon T, Mel B (2003) Pyramidal neuron as 2-layer neural network. Neuron 37:989-999

Van den Pol A, Obrietan K, Belousov A (1996) Glutammate hyperexcitability and seizure-like activity throughout the brain and spinal cord upon relef from chronic glutammate receptor blockade in culture. Neuroscience 74:653-674

Polsky A, Mel B, Schiller J (2004) Computational subunits in thin dendrites of pyramidal cells. Nat Rev Neurosci 7(6):621-627

Potter S, DeMarse T (2001) A new approach to neuronal cell culture for long-term studies. J Neurosci Meth 59:782-806

Powers R, Sawczuk A, Musick J, Binder M (1999) Multiple mechanisms of spike-frequency adaptation in motoneurones. J Physiol (Paris) 93:101-114

Prescott S, De Koninck Y (2003) Gain control of firing rate by shunting inhibition: eoles of synaptic noise and dendritic saturation. PNAS 100:2076-2081

Rall W (1967) Distinguishing theoretical synaptic potentials computed for different soma-dendritic distributions of synaptic input. J Neurophysiol 30(5):1138-1168

Renart A, Brunel N, Wang XJ (2003) Mean-field theory of recurrent cortical networks: from irregularly spiking neurons to working memory. In: Feng J (ed) Computational neuroscience: a comprehensive approach. CRC Press, Boca Raton

Richardson MJE, Gerstner W (2005) Synaptic shot noise and conductance fluctuations affect the membrane voltage with equal significance. Neural Comput 17:923-947

van Rossum M, Turrigiano G, Nelson S (2002) Fast propagation of firing rates through layered networks of noisy neurons. J Neurosci 22:1956-1966 
Sah P (1996) $\mathrm{Ca}^{2+}$-activated $\mathrm{K}^{+}$currents in neurons: types, physiological roles and modulation. Trends Neurosci 19:150-154

Salinas E, Thier P (2000) Gain modulationa major computational principle of the central nervous system. Neuron 27:15-21

Schwindt P, O'Brien J, Crill W (1997) Quantitative analysis of firing properties of pyramidal neurons from layer 5 of rat sensorimotor cortex. J Neurophysiol 77:2484-2498

Segev I, London M (2000) Untangling dendrites with quantitative models. Science 290(5492):744-750

Sherman S, Guillery R (1998) On the actions that one nerve cell can have on another: distinguishing "drivers". Proc Natl Acad Sci USA 95:7121-7126

Shriki O, Hansel D, Sompolinsky H (2003) Rate models for conductance-based cortical neural networks. Neural Comput 15:1809-1841

Spruston N (2008) Pyramidal neurons: dendritic structure and synaptic integration. Nat Rev Neurosci 9(3):206-221

Thorson J, Biederman-Thorson M (1974) Distributed relaxation processes in sensory adaptation. Science 183:161-172

Thurley K, Senn W, Lscher H (2008) Dopamine increases the gain of the input-output response of rat prefrontal pyramidal neurons. J Neurophysiol 99(6):2985-2997
Treves A (1993) Mean field analysis of neuronal spike dynamics. Network 4:259-284

Tuckwell HC (1988) Introduction to theoretical neurobiology. Cambridge University Press, Cambridge

Ulanovsky N, Las L, Farkas D, Nelken I (2004) Multiple time scales of adaptation in auditory cortex neurons. J Neurosci 24:10,44010,453

van Vreeswijk C, Hansel D (2001) Patterns of synchrony in neural networks with spike adaptation. Neural Comput 13:959-992

Wagenaar D, Pine J, Potter S (2006) An extremely rich repertoire of bursting patterns during the development of cortical cultures. BMC Neurosci 7:11

Wark B, Lundstrom B, Fairhall A (2007) Sensory adaptation. Curr Opin Neurobiol 17:423-429

Xu Z, Payne J, Nelson M (1996) Logarithmic time course of sensory adaptation in electrosensory afferent nerve fibers in a weakly electric fish. J Neurophysiol 47:2020-2032

Zhang Z, Arsenault D (2005) Gain modulation by serotonin in pyramidal neurones of the rat prefrontal cortex. J Physiol 566(2):379-394 